\title{
editorial
}

\section{Las aportaciones del programa de la Especialidad de Medicina Familiar y Comunitaria}

\begin{abstract}
$\mathscr{P}$ rom obablemente el nuevo programa de la Especialidad de Medicina Familiar y Comunitaria, aprobado el 20 de noviembre de 2002 por la Comisión Nacional de la Especialidad (CNE) y presentado el 22 de noviembre en Madrid en el XXII Congreso Nacional de la semFYC, sea conocido y reconocido, fundamentalmente, por su propuesta de incremento de tiempo a cuatro años. Pero es algo más. Creemos firmemente que es mucho más.

Hasta ahora, todos los programas de la Especialidad han propuesto avances decisivos y, junto con otras aportaciones, han ido conformando el perfil profesional y el cuerpo doctrinal de nuestra especialidad. Y este programa no podía ser menos.

Antes de analizar sus aportaciones específicas, es necesario hacer una valoración de su potencialidad, que se basa en dos hechos. El primero es el grado de consenso y de participación alcanzados en su desarrollo, planteamiento, elaboración y defensa. El segundo es la metodología empleada.
\end{abstract}

Este programa inició su andadura hace tres años y ha pasado por diferentes fases: análisis de la situación, diseño del programa, elaboración y consenso, como etapas previas a la fase de ejecución, que deseamos sea realidad en la convocatoria MIR del año 2004.

El análisis de la situación se llevó a cabo mediante búsqueda de consenso con el método Delphi. En él participaron coordinadores de Unidades Docentes (UUDD), tutores y residentes, identificando las fuerzas y debilidades del actual programa y las líneas futuras de trabajo. De ese trabajo se extrajo el análisis de situación, no sólo del programa en sí, sino también de los condicionantes sociales y sanitarios actuales y de los previsibles en el futuro. De forma paralela, se realizó una revisión de los programas de medicina de familia en el ámbito internacional, revisando casi un centenar de ellos, fundamentalmente los de varias Universidades de los EE.UU., Canadá, Finlandia, Suecia, Dinamarca, Reino Unido, entre otras. Teniendo en cuenta que se ha tenido siempre presente la absoluta necesidad de elaborar un programa con el máximo grado de consenso y de participación posible, se vió la oportunidad de establecer un grupo de coordinación editorial y de redacción del nuevo programa en el que estuviera representada la CNE, la estructura docente (con la participación de coordinadores, tutores y residentes) y la semFYC en su calidad de sociedad científica con mayor grado de representatividad en Atención Primaria. Este grupo tuvo el encargo específico de perfilar y diseñar el formato del programa y las líneas maestras del mismo. Una vez alcanzado este 
consenso se encargó a grupos de expertos el desarrollo de los diferentes apartados del programa, participando un total de 82 redactores. Efectuado este trabajo, el grupo coordinador se ocupó de homogeneizar los diferentes contenidos, para finalmente remitir el borrador a todas las UUDD de las 17 Comunidades Autónomas, a la semFYC y a sus sociedades federadas y a la SEMERGEN. En este proceso de corrección han participado 154 correctores aportando importantes sugerencias para la mejora de la calidad del programa. En definitiva, más de 400 folios de sugerencias, que fueron analizadas e incorporadas en su mayor parte.

Se ha alcanzado, por tanto, un elevado grado de consenso, consecuencia del altísimo grado de implicación y participación en el programa. Ha participado la CNE, ha participado la semFYC a través del apoyo logístico, facilitando las reuniones del grupo de trabajo así como el contacto con los diferentes expertos que trabajan en los grupos semFYC, han participado muchos coordinadores, tutores y residentes. En definitiva, se puede afirmar que éste es un proyecto de todos nosotros, de los médicos de familia.

\section{LAS APORTACIONES}

Las principales aportaciones del programa son las siguientes:

1. La metodología. Tanto el diseño del programa en sí, ya comentado, como la metodología formativa propuesta están en consonancia con las nuevas tendencias pedagógicas. La solidez metodológica alcanzada da validez al programa para convertirse en un referente en metodología formativa de postgrado para el resto de especialidades.

El programa parte del análisis de situación, enmarca los valores profesionales del médico de familia y redefine el perfil profesional y, con este marco, diseña las competencias del médico de familia. En cada área competencial se definen los objetivos a conseguir y las actividades propias para obtener los conocimientos, habilidades y actitudes; asimismo, se determinan la metodología docente, los lugares de aprendizaje, el tiempo de aprendizaje, la evaluación y la bibliografía recomendados.

Existen tres elementos novedosos en el programa que deben ser reseñados:

- La definición de los niveles de prioridad. Se definen tres niveles de prioridad. Prioridad I: las competencias son consideradas indispensables y deben ser adquiridas por todos los residentes, su ausencia cuestiona su aptitud. Prioridad II: las competencias son importantes y deben ser adquiridas por la mayoría de los residentes. Y prioridad III: la adquisición de estas competencias, si lo anterior está presente, puede ser un criterio de excelencia.

- La definición de los niveles de responsabilidad. Tres niveles. Nivel primario: el médico de familia debe ser capaz de identificar, evaluar y 
tratar este tipo de problemas sin consultar con otro nivel asistencial en el 90\% de los casos. Nivel secundario: una consulta a otro nivel es habitualmente requerida en el curso de la evaluación o del tratamiento de este tipo de problemas. Y nivel terciario: el diagnóstico y tratamiento de estos problemas es competencia de otros especialistas, pero el médico de familia debe ser capaz de informar, de apoyar al paciente y a la familia y de asegurar la coordinación y la continuidad de la atención.

Esta definición no sólo representa un elemento en metodología docente sino que además permite avanzar en el perfil competencial del médico de familia y profundiza en el análisis de los límites y de las relaciones con otras especialidades ayudando a conformar un elemento importante en la relación entre niveles como son los criterios de derivación.

- Los métodos formativos propuestos. Se considera en todo momento la flexibilidad como cualidad intrínseca del programa, en tanto en cuanto lo importante son las competencias que van a adquirirse, entendiendo que los métodos pueden diferir según las unidades docentes, aunque en principio todos ellos son complementarios. Los básicos son el autoaprendizaje dirigido y el aprendizaje de campo, que puede completarse con los cursos, los talleres y trabajo en grupo, según se trate de adquirir conocimientos nuevos, aprehender habilidades o mejorar las actitudes.

La relación tutor-residente es el elemento y eje fundamental de la formación, ya que la tutorización personalizada, continuada y estructurada es el método básico de aprendizaje. Incorpora en este concepto la evaluación formativa, es decir, el seguimiento de la adquisición de competencias. Y por esto, y con relación a esto, se incorporan la ampliación de entornos docentes y las estancias electi vas de tres meses al final del tercer año, con el objeto de adquirir o mejorar competencias en las que el residente no haya alcanzado el nivel adecuado.

Por otra parte, esto presupone el apoyo a los tutores para el mantenimiento y mejora de sus niveles competenciales, tanto en las áreas propias del programa como en metodologías docentes, y para que las condiciones estructurales de trabajo sean las adecuadas para la realización del trabajo docente.

2. Los valores profesionales y el perfil profesional. En una época en la que el burn-out y la medicina defensiva hacen acto de presencia es importante recordar los valores profesionales del médico de familia, que están vinculados a su compromiso con las personas, su compromiso social y ético, su compromiso con la mejora continua, con la propia especialidad y con la formación a largo plazo. Los tutores no sólo debemos favorecer el aprendizaje de conocimientos, habilidades y actitudes sino que debemos transmitir a los residentes los valores positivos de nuestra profesión. 
En el nuevo programa, el perfil profesional se redefine y se consideran algunas áreas, hasta ahora pensadas como áreas de apoyo, cómo áreas esenciales en el quehacer del médico de familia. De este modo, se reubica la importancia de áreas como la comunicación, el razonamiento clínico, la bioética y la gestión de la atención (gestión clínica, trabajo en equipo, gestión y organización de la actividad, sistemas de información, gestión de la calidad y responsabilidad civil y médico-legal). La cercanía de la medicina de familia a la realidad social y su importancia en el sistema sanitario hacen que se profundice en las áreas propias de la medicina de familia, como la Atención a la Familia y la Atención a la Comunidad y la Atención al Individuo en sus dos aspectos de abordaje de necesidades y problemas de salud, como de abordaje de grupos poblacionales y de grupos con factores de riesgo. Esto conlleva la incorporación de áreas nuevas como son la atención a conductas de riesgo, la atención a urgencias y emergencias, la atención a las personas en situaciones de riesgo familiar (violencia doméstica) o en situaciones de riesgo social (la exclusión social y los discapacitados). Esta presencia en el programa confiere pluripotencialidad al mismo. No sólo se forman médicos para que trabajen en Atención Primaria sino también para que puedan trabajar en entornos donde el perfil profesional del médico de familia pueda ser el adecuado, como son las urgencias o emergencias u otras unidades o servicios que pueden servir de apoyo a la Atención Primaria. También puede servir de base para la definición de posibles áreas de capacitación a desarrollar después de la formación postgrado.

El compromiso con la formación a largo plazo hace que ésta se incorpore como área propia del programa ya que se considera el programa como una herramienta fundamental no sólo para la formación postgrado sino para la formación continuada del médico de familia. Es decir, este programa no sólo tiene interés para los tutores y residentes sino para todos los médicos de familia, al convertirse en un referente de manteni miento de la competencia en medicina de familia y comunitaria.

En un momento como el actual, con las transferencias sanitarias a todas las comunidades autónomas y con la probable rápida publicación de la Ley de Cohesión y Calidad del Sistema, este programa, por su definición de un posible catálogo de servicios de la Atención Primaria y su concordancia con el anteproyecto de la referida ley, se convierte en una potente herramienta para la cohesión y calidad del sistema sanitario.

3. El tiempo. Como se decía en un inicio, el tiempo de formación probablemente sea el elemento más recordado de este programa. Su ampliación a cuatro años se fundamenta, por una parte, sobre la realidad actual. El programa actual está conllevando más de tres años si contabilizamos los tiempos de guardias y los tiempos de formación teóricopráctica que se efectúan fuera del horario laboral. Pero, por otra parte, si se suman los tiempos mínimos recomendados para el aprendizaje de cada área competencial se llega a la conclusión de la inviabilidad de la ad- 
quisición de competencias en menos de cuatro años. Esto, además, responde a lo solicitado por la Unión de Médicos Generales de Europa (UEMO) al Parlamento Europeo de un incremento de tiempo formativo específico de formación en medicina de familia de cinco años, emulando los programas más avanzados de Austria, Dinamarca o Finlandia.

Todo esto se enmarca, no sólo en la defensa de la especialidad, sino en el comprobado impacto sobre los sistemas sanitarios en términos de equidad, efectividad y eficiencia que tiene una Atención Primaria con médicos de familia bien formados. Es decir, se enmarca básicamente en nuestro compromiso social y nuestro compromiso con las personas y su salud.

\section{AGRADECIMIENTOS}

A todos los que han hecho posible la realización del programa y, sobre todo, a todos los que van a hacer posible su desarrollo. A Luis y Antonio por sus sugerencias.

V. CASADO VICENTE

Coordinadora del Grupo Editorial y de Redacción del Programa de la Especialidad de Medicina Familiar y Comunitaria

\section{BIBLIOGRAFÍA}

1. Comisión Nacional de la Especialidad de Medicina Familiar y Comunitaria. Programa de la Especialidad de Medicina Familiar y Comunitaria (aprobado el 20-XI-2002). Disponible en Web: http:// www.semfyc.es. 